Trauma Surgery $\&$ Acute Care Open

\section{Blunt rupture of the thoracic duct after severe thoracic trauma}

Department of Surgery, CHI Health Creighton University Medical Center Bergan Mercy, Omaha, Nebraska, USA

Correspondence to Dr Juan Antonio Asensio, Department of Surgery, CHI Health Creighton University Medical Center Bergan Mercy, Omaha, NE 68124, USA; juanasensio@creighton.edu

Received 26 March 2018 Accepted 30 May 2018
To cite: Brown SR, Fernandez C, Bertellotti R, et al. Trauma Surg Acute Care Open 2018;3:e000183.
A 53-year-old man was admitted to our trauma center after sustaining thoracoabdominal injuries, secondary to a rear-end motor vehicle collision. As he stepped out of his vehicle, he was struck by a tractor trailer at $55 \mathrm{mph}$. The following were the initial vital signs on his arrival: heart rate 140 beats/min, blood pressure $142 / 80 \mathrm{~mm} \mathrm{Hg}$, respiratory rate 28 breaths $/ \mathrm{min}$, temperature $36.8^{\circ} \mathrm{C}$, and oxygen saturation 93\%. The Glasgow Coma Scale score was 15 and the Injury Severity Score was 59 . He was evaluated and resuscitated per the advanced trauma life support protocols. The focused assessment with sonography for trauma examination was negative. Initial findings included bilateral chest wall and thoracic spine tenderness, subcutaneous emphysema in the chest and neck, and an unstable pelvis. He required bilateral chest tubes and a pelvic binder. CT imaging revealed a left temporal epidural hematoma, multiple facial fractures, a sternal fracture, a left scapula fracture, acromioclavicular fractures, bilateral hemopneumothoraces, pulmonary contusions, extensive pneumomediastinum compressing the right atrium, multiple rib fractures (2-10 on the left with a flail segment and 2-8 on the right) (figure 1), an unstable open-book pelvic fracture which included bilateral superior and inferior pubic rami fractures, sacral and left iliac wing fractures, and symphysis pubis diastasis.

The patient developed hypotension and severe respiratory distress, and was intubated. ECG revealed no dysrhythmias. Echocardiogram revealed significant left ventricular wall dysfunction

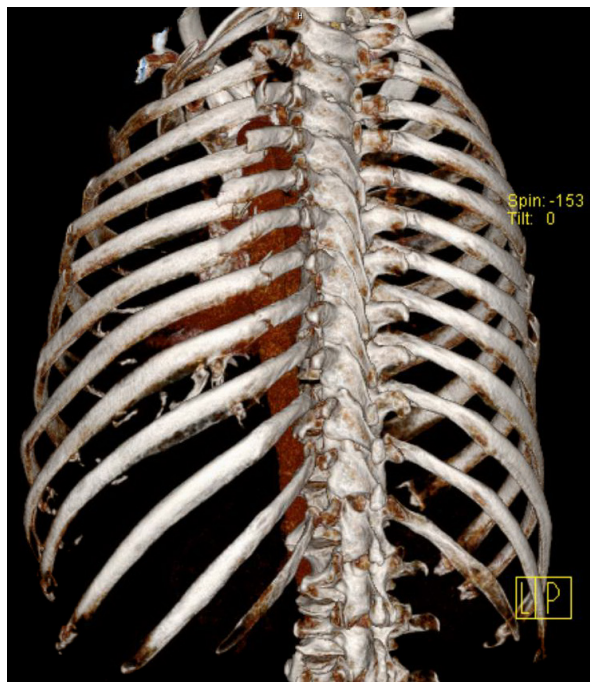

Figure 1 Three-dimensional CT scan reconstruction demonstrating left-sided flail chest.

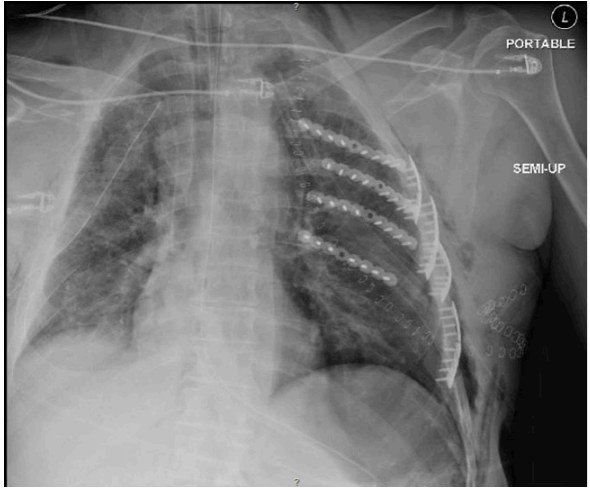

Figure 2 Postoperative chest X-ray demonstrating left chest wall reconstruction.

consistent with myocardial contusion and right atria compression. His troponins were also significantly elevated. He required significant resuscitation with crystalloids, blood products and vasopressors. He underwent bronchoscopy, esophagram and upper endoscopy to exclude tracheoesophageal injury, and these were negative. On hospital day 2, the patient was hemodynamically stable, and pressors were discontinued. His pelvic fractures were repaired using external fixation and sacral screws. Given his extensive left flail chest, he underwent reconstruction of his left chest wall on hospital day 5. Open reduction and internal fixation of his left ribs, 3 to 6 anteriorly and 4 to 7 posteriorly, with titanium plates was performed (figure 2). He had an epidural catheter inserted for analgesia. On postoperative day 2 after chest wall reconstruction, the patient was extubated and resumed enteral feeds. Overnight, the output from the left-sided chest tube changed from serosanguinous to milky. A sample was sent for triglycerides and lymphocyte counts confirming the diagnosis of chylothorax. His chest tube output increased to approximately $2000 \mathrm{~mL} /$ day. A lymphangiogram was performed with Lipiodol to diagnose the location of the chylous leak. It revealed contrast extravasation at the level of T3 to T4. An MRI was also performed to better define the anatomic course of the thoracic duct.

\section{WHAT WOULD YOU DO?}

A. Conservative management: placing the patient nulla per os (NPO), and starting total parentera nutrition (TPN), octreotide and midodrine.

B. Thoracic duct embolization by interventional radiology.

C. CT-guided thoracic duct disruption.

D. Thoracotomy with thoracic duct ligation. 


\section{WHAT WE DID AND WHY}

\section{Correct answer: D}

We initially attempted all of these interventions as a trial of non-operative and minimally invasive management given that the patient had recently undergone extensive left chest wall reconstruction, and was at an increased risk for titanium plate infection and empyema. We also wanted to prevent disruption of the chest wall reconstruction.

The patient was made NPO, placed on TPN, and started on octreotide and midodrine. Interventional radiology attempted to perform a thoracic duct embolization via lymphangiography. This was unsuccessful. Interventional radiology also performed a CT-guided thoracic duct disruption in which they attempted to macerate the thoracic duct and cisterna chyli with several passes of a large bore needle. This combination of interventions reduced the chylothorax output from 2000 to $1000 \mathrm{~mL} /$ day, but it remained high output.

Ultimately, the patient required a left posterolateral thoracotomy, decortication, thoracic duct ligation and pleurodesis to control his chylothorax. The patient's chest tube output had become serous in nature while he was kept NPO for his trial of conservative management, so olive oil and $60 \mathrm{~mL}$ of cream were administered through a nasogastric tube at the time of skin incision for the thoracotomy to stimulate the flow of chyle and aid in the identification of the leak. Once we had gained entrance into the left pleural cavity and completed our decortication, the thoracic duct was identified near Poirier's triangle, adjacent to the junction of the subclavian artery and aortic arch. Selective suture ligation and clip placement was then used to control the leak (figure 3).

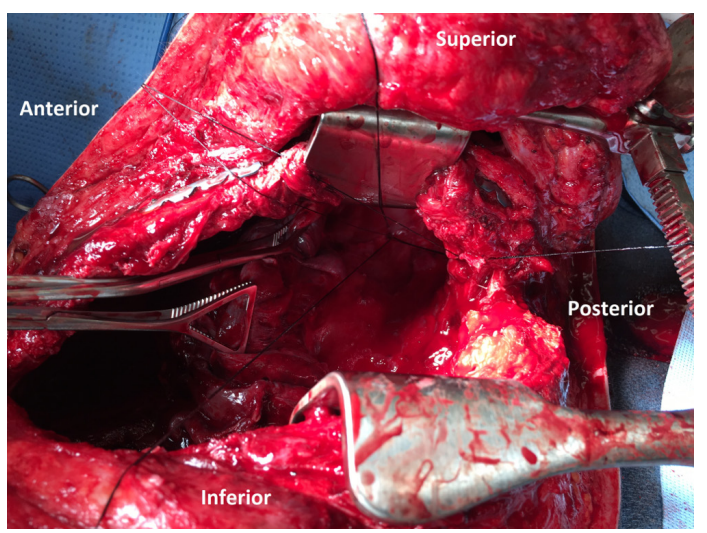

Figure 3 Ligated thoracic duct.

Postoperatively, the patient's chest tube output decreased precipitously and became serosanguinous in nature. Fluid triglyceride and lymphocyte counts showed resolution of the chylothorax. We wanted to keep the patient in a state of low chylous flow while his thoracic duct stump healed, so he remained NPO and on TPN for 2 weeks prior to initiating a diet. His diet was advanced without any recurrence of the chylothorax and his chest tubes were removed. He was discharged to a rehabilitation facility. Currently, the patient has normal chest X-rays and CT scans of the thorax. He has been followed postoperatively for 6 months without any recurrence. 